OPEN ACCESS

Understanding Electrical Under- and Overshoots in Proton Exchange Membrane Water Electrolysis Cells

To cite this article: C. Immerz et al 2019 J. Electrochem. Soc. 166 F1200

View the article online for updates and enhancements. 


\title{
Understanding Electrical Under- and Overshoots in Proton Exchange Membrane Water Electrolysis Cells
}

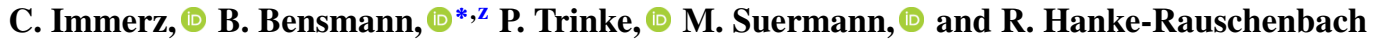 \\ Institute of Electric Power Systems, Leibniz Universität Hannover, 30167 Hannover, Germany
}

\begin{abstract}
Ability of dynamic operation seems to be an important feature of proton exchange membrane water electrolyzers (PEMWE) to become a relevant part of the future energy system. However, only few fundamental analyzes of the dynamic behavior on short time scales are available in the literature. Therefore, this contribution aims to give insights into the most fundamental transient behavior of a PEMWE cell by an experimental analysis on the laboratory scale and a model based description of the ongoing phenomena. Experimental voltage and current controlled load step are carried out and analyzed by methods adapted from fuel cell characterization. The experimental analysis revealed that load steps are a combination of an instantaneous characteristic followed by dynamics of higher order dependent on activation, mass transfer and temperature effects. Potentiostatic downward steps to very low cell voltages can lead to current density reversal phenomena with highly negative peak current densities. By means of a simple prototype model analysis, these reversal processes are analyzed and the consequences of the phenomena are estimated. The simulation results indicate that a reversal of the cell current density can be attributed to a change of capacitive rather than faradaic currents, meaning that internal electrolysis processes are not involved.

(C) The Author(s) 2019. Published by ECS. This is an open access article distributed under the terms of the Creative Commons Attribution 4.0 License (CC BY, http://creativecommons.org/licenses/by/4.0/), which permits unrestricted reuse of the work in any medium, provided the original work is properly cited. [DOI: $10.1149 / 2.0881914$ jes]

(cc) BY
\end{abstract}

Manuscript submitted July 17, 2019; revised manuscript received September 19, 2019. Published November 5, 2019.

Although its important role in future energy systems is widely discussed, the broad market penetration of water electrolysis suffers from too high specific hydrogen production costs. Besides reduction of capital and operational expenditures, additional revenues, e.g. by providing ancillary services, could lower the hydrogen production cost.

Dynamic operation offers an opportunity for such a service extension of water electrolysis systems and in particular for proton exchange membrane water electrolysis (PEMWE) systems. Several studies analyzed the theoretical possibilities for PEMWE systems to participate in the grid balancing market ${ }^{1}$ or have calculated economic and technological system opportunities for existing systems. ${ }^{2}$ Furthermore, first demonstration plants in the megawatt scale are recently in operation and will provide insights in the dynamic plant operation in the near future. ${ }^{3,4}$ However, there are still various challenges in a dynamic operation mode that need to be understood for scientific but also for technical reasons, as summarized by Babic et al. ${ }^{5}$

An essential aspect is the dynamic behavior of the electrolyzer itself. This topic is so far addressed in few modeling studies ${ }^{6-8}$ and some experimental works. ${ }^{9,10}$ The analyzes of Olivier et al. ${ }^{6}$ and Guilbert et al. ${ }^{10}$ mainly focused on the thermodynamic behavior of stacks. Görgün ${ }^{7}$ and Yigit et al. ${ }^{8}$ focused on the changes in hydrogen production rates with system load changes on time scales of several minutes or longer, while Eichmann et al. ${ }^{9}$ was tracking the electrical responses of stacks on time scales of seconds and below.

In contrast, the present work focuses on a more fundamental analysis, which is inspired from proton exchange membrane fuel cell (PEMFC) research. Load step analyzes for fuel cells revealed that an instantaneous characteristic is typical followed by a highly complex transient behavior due to activation, concentration and temperature effects. ${ }^{1-13}$ Local analyses of the electrical and electrochemical response behavior have shown significant differences at the inlet and the outlet of a cell ${ }^{14}$ and have indicated critical dynamic operation modes. ${ }^{15}$ Additionally, unfeasible load steps are reported, which can be characterized by the reversal curve. ${ }^{13}$ The results from fuel cell research have shown that the electrical response to simple load steps can be considered as the most elementary part of a dynamically operated electrochemical cell.

Therefore, in this work, experimental load steps are performed to figure out similarities with the PEMFC literature in order to understand the related mechanisms in PEMWE cells. The proven PEMFC methods are transferred to the analysis of the electrolysis cell with

*Electrochemical Society Member.

${ }^{\mathrm{z}}$ E-mail: boris.bensmann@ifes.uni-hannover.de special focus on the instantaneous characteristic and the reversal curve.

\section{Experimental}

Experimental load steps were performed with a PEMWE cell of laboratory scale. The cell, the test station and the measurement procedure are described in the following section.

Cell setup.-A square $4.0 \mathrm{~cm}^{2}$ PEMWE cell from balticFuelCells $\mathrm{GmbH}$ (qCf FC25/100 V 1.1) with integrated electrical temperature control is used. The cell has two titanium coated flow fields with 13 parallel channels each on the anode and cathode. Both half-cells are sealed with O-ring gaskets.

The cell is equipped with a sintered titanium porous transport layer (PTL) on the anode (mott corp., nominal thickness: $1000 \mu \mathrm{m}$, particle diameter: $\approx 50 \mu \mathrm{m}$, porosity: $40 \%$ ). On the cathode side, a carbon paper (Freudenberg Performance Materials SE \& Co. KG, H23I2, nominal thickness: $222 \mu \mathrm{m}$ ) is used as PTL.

A commercial catalyst coated membrane (CCM) from HIAT $g G m b H$ is used. The CCM (MEA E N117 $5 \mathrm{~cm}^{2}$ ) is a Nafion 117 membrane coated with an Ir-black catalyst on the anode side (loading: $2 \mathrm{mg}_{\mathrm{lr}} / \mathrm{cm}^{2}$ ) and a $\mathrm{Pt} / \mathrm{C}$ catalyst on the cathode side (loading: $1 \mathrm{mg}_{\mathrm{Pt}} / \mathrm{cm}^{2}$ ).

Test station.- - The experiments are carried out on a fully integrated test station from Greenlight Innovation. The anode water flux is set to $20 \mathrm{~mL} \mathrm{~min}{ }^{-1} \mathrm{~cm}^{-2}$ at a temperature of $60^{\circ} \mathrm{C}$ and ambient pressure. The electric conductivity of the feed water is below $1.0 \mu \mathrm{S} \mathrm{cm} \mathrm{cm}^{-1}$. Furthermore, the electrical temperature control of the Baltic test cell is also set to $60^{\circ} \mathrm{C}$. Prior to the experiments the cell is purged with nitrogen on the anode and cathode. During the experiments the cathode inlet is closed.

The electrical supply and the response analysis are realized with a galvanostat/potentiostat from Bio-Logic Science Instruments (SP-150) with external power booster for measurements up to 20A(VMP3B-20). The galvanostat/potentiostat's fast data acquisition of the electrical data of $0.2 \mathrm{~ms}$ is used in order to measure fast current or voltage controlled load steps. The control speed of the galvanostat/potentiostat is fast so that in less then $0.6 \mathrm{~ms}$ the difference between the starting and end point is reached by about $99.5 \%$. The control of the galvanostat/potentiostat and the electrical data acquisition are performed with the software EC-lab from Bio-Logic. 
(a) Galvanostatic

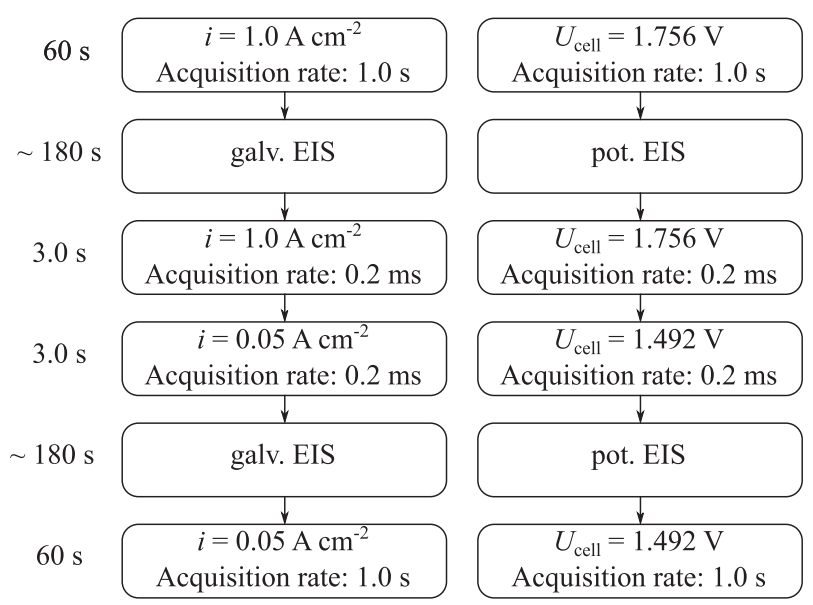

Figure 1. Exemplary load step procedure for galvanostatic (a) and potentiostatic (b) downward step from $1.0 \mathrm{~A} \mathrm{~cm}^{-2}$ to $0.05 \mathrm{~A} \mathrm{~cm}^{-2}$ (resp. $1.756 \mathrm{~V}$ to $1.492 \mathrm{~V})$.

Measurement protocol.-The measurement protocol is split into the general pre- and post-testing of the cell and the dynamic load step experiments. For the general testing, galvanostatic polarization curves are recorded at the beginning and the end of the tests in the current density range from 0.001 to $3.0 \mathrm{~A} \mathrm{~cm}^{-2}$ with holding times of $10 \mathrm{~s}$ per step. The high frequency resistance (HFR) is measured at each current density step by a single-frequency impedance measurement at $15.2 \mathrm{kHz}$, which includes all pure ohmic resistances of the cell. ${ }^{16}$ Subsequently, electrochemical impedance spectroscopy (EIS) is performed in the frequency range from $100 \mathrm{kHz}$ to $0.1 \mathrm{~Hz}$ to countercheck, if the low frequency resistance is similar to the slope of the previously measured polarization curve. ${ }^{17}$ Both, the HFR measurement and the full impedance spectra are recorded in a galvanostatic mode with a perturbation amplitude of $10 \%$ of the adjusted DC current.

As aforementioned, the transient behavior of the galvanostatically and potentiostatically controlled load steps is investigated. Therefore, the identical measurement setup is used. A starting current density (resp. cell voltage) is adjusted and held for $60 \mathrm{~s}$ at steady-state conditions. Afterwards, an EIS is performed to distinguish between possible loss changes. The starting value is held for $3 \mathrm{~s}$ with the highest data acquisition time (i.e. $0.2 \mathrm{~ms}$ ), followed by the load step. The new set point is held for another $3 \mathrm{~s}$ still with the same data acquisition rate. Then, a second EIS is performed, followed by a period of a constant current density (resp. cell voltage) of $60 \mathrm{~s}$. The perturbation amplitude is $10 \%$ of the adjusted DC current for the galvanostatic EIS and a fixed voltage amplitude of $10 \mathrm{mV}$ for potentiostatic EIS measurements. The overall procedure for each load step experiment sums up to approximately $500 \mathrm{~s}$ (see Fig. 1). Table I summarizes the voltage, current density and power density pairs of the performed steps.

Table I. Experimentally performed load steps. Steady state pairs of the cell voltage, current density and power density.

\begin{tabular}{lccc} 
Step & $i / \mathrm{A} \mathrm{cm}^{-2}$ & $U_{\text {cell }} / \mathrm{V}$ & $p / \mathrm{W} \mathrm{cm}^{-2}$ \\
\hline Start & 1.00 & 1.756 & 1.756 \\
down 1 & 0.01 & 1.451 & 0.015 \\
down 2 & 0.05 & 1.492 & 0.075 \\
up 1 & 2.00 & 1.983 & 3.966 \\
up 2 & 3.00 & 2.204 & 6.612
\end{tabular}

\section{Results}

In the following section the experimental results of current controlled load steps (galvanostatic) are presented, followed by the results for load steps in a voltage controlled mode (potentiostatic) according to Table I. Transferred to the technical systems, the selected cases represent load steps over a wide range of the operating window. For instance, assuming a current density of $1.0 \mathrm{~A} \mathrm{~cm}^{-2}$ to be the corresponding nominal operation point, herein referred to as $100 \%$, upward load steps to $200 \%$ and $300 \%$ on the one side, and downward load steps to $1.0 \%$ and $5.0 \%$ on the other side are investigated.

Galvanostatic steps.-Fig. 2a shows the current density inputs for the experimentally performed galvanostatic load steps over time. All performed steps start at a current density of $i=1.0 \mathrm{~A} \mathrm{~cm}^{-2}$. Two upward steps (blue circle and red diamond) and two downward steps (green triangle and purple square) to different current densities are performed. Independent of the relatively large load steps, the galvanostat/potentiostat reaches both the upward and the downward current density set-points within less then $1.0 \mathrm{~ms}$, which means that the control quality of the galvanostat/potentiostat is correspondingly fast. For more details on control speed and accuracy, please check Test station section.

The voltage responses on the four current density load steps are shown in Fig. 2b on the identical linear time scale as the input signals. The voltage responses show an initial step in the moment of the load step, followed by a transient run-in into the new steady state condition. At this scale, the trends show either monotonously rising progressions for upward load steps or monotonously decreasing progressions for downward steps. No voltage over- or undershoots are observed.

To gain a deeper insight in the dynamic behavior, the four insets (Figs. 2b-1-2b-4) show the cell voltage responses on logarithmic time scales directly after the initial response. The upward steps, shown in insets Fig. 2b-1 and Fig. 2b-2, reveal dynamics of higher orders, indicated by the two inflection points of the curves. Higher order dynamics may also be possible for downward steps, however such behavior cannot be observed from the present data (s. insets Fig. 2b-3 and Fig. $2 \mathrm{~b}-4)$. All voltage responses show that the new steady state conditions are reached within $3 \mathrm{~s}$ after each load step.

Fig. 2c shows the input current density $(i(t))$ and the output cell voltage $\left(U_{\text {cell }}(t)\right)$ projected into the two-dimensional polarization space, $U_{\text {cell }}$ vs. $i$. The time-dependent current density and cell voltage pairs form the trajectories of the steps. Additionally, the steady state polarization curve is shown (black dots), on which the vertical, thin lines indicate the start and end current densities. The dashed line $\left(U_{\text {init }}(i)\right)$ is calculated as a linear equation with the specific ohmic resistance at the starting current density as slope $\left(r_{\mathrm{HFR}}\left(i=1.0 \mathrm{~A} \mathrm{~cm}^{-2}\right)\right.$ $=190 \mathrm{~m} \Omega \mathrm{cm}^{2}$ ).

$$
U_{\text {init }}(i)=r_{\mathrm{HFR}} \cdot i+U_{\mathrm{x}} .
$$

This line is shifted parallel in such a way that it intersects the polarization curve at the starting point. The intersection of this curve with the ordinate at $i=0 \mathrm{~A} \mathrm{~cm}^{-2}$ is defined as $U_{\mathrm{x}}$.

Potentiostatic steps.-For the potentiostatic load steps, set cell voltages are selected similar to the corresponding cell voltages obtained by the aforementioned galvanostatic experiments. Similar to the current controlled load steps, the input voltage signals for potentiostatic steps are presented in Fig. 3a based on Table I. Two upward and two downward steps are performed, each with a starting cell voltage of $U_{\text {cell }}=1.756 \mathrm{~V}$. Analogous to the galvanostatic step, the galvanostat/potentiostat reaches the new cell voltage set-points for upward and downward steps within less than $1.0 \mathrm{~ms}$, with a similar control quality compared to the aforementioned galvanostatic step (see Galvanostatic steps, Test station sections).

Analogous to before, Fig. $3 \mathrm{~b}$ presents the current density responses of the four potentiostatic steps on a linear time scale. In comparison to the galvanostatic load steps, the potentiostatic steps do not show monotonous response behavior. Instead, the upward responses (blue 

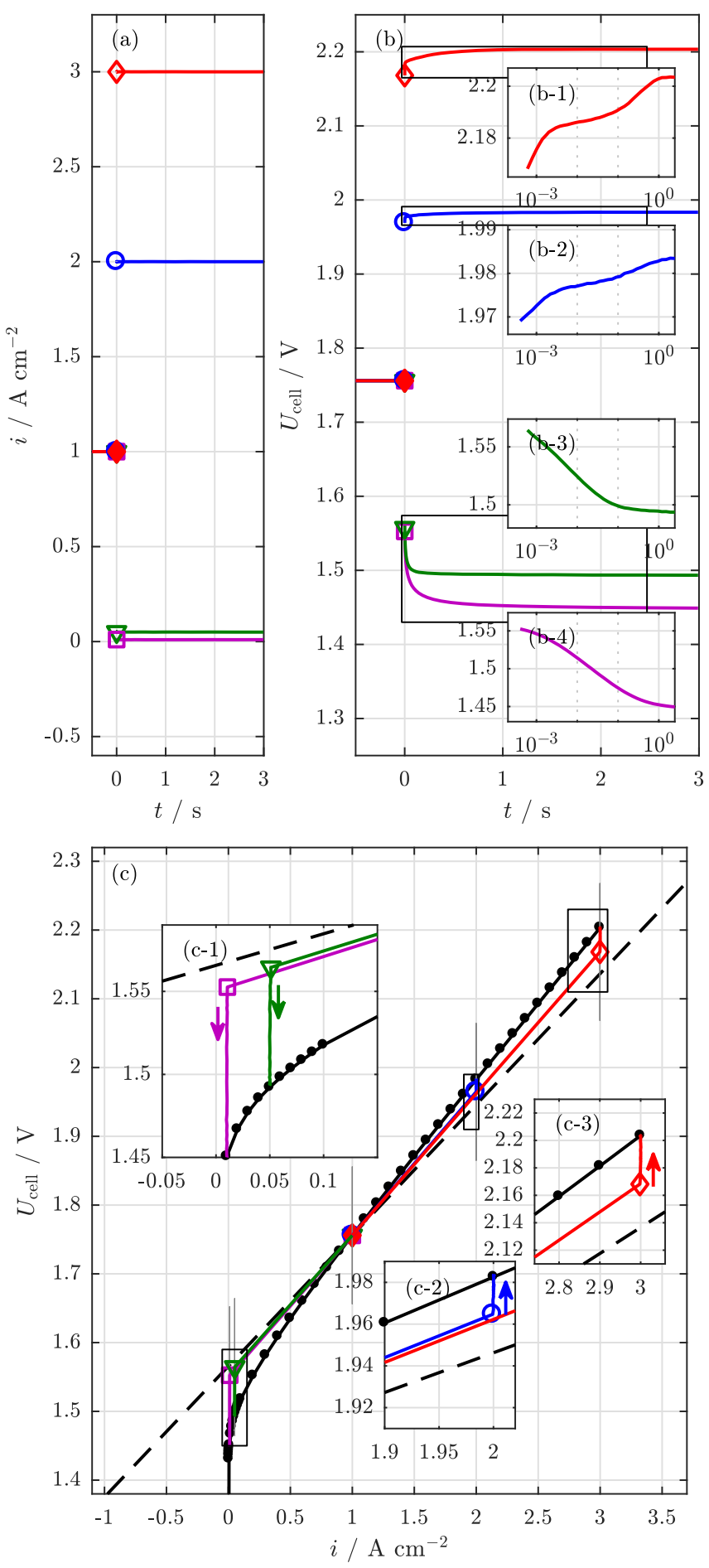

Figure 2. Current controlled upward and downward load steps; (a) current density input step, full markers: start value, open markers: end value; (b) cell voltage responses; Insets (b-1)-(b-4): different cell voltage responses on a logarithmic time-scale. (c) steady state polarization curve (black dots) with trajectories of the four load steps in the Ui-phase space (with markers according to (a)).

circle, red diamond) show strong overshoots, while the downward responses (violet square, green triangle) undershoot initially. After the initial peak, the current density runs-in in the new steady state within 3 s monotonously.

The run-in of the current density responses is presented in more detail in the insets Figs. 3b-1-3b-4 on a logarithmic time scale. The current density responses also show trends with at least two inflection
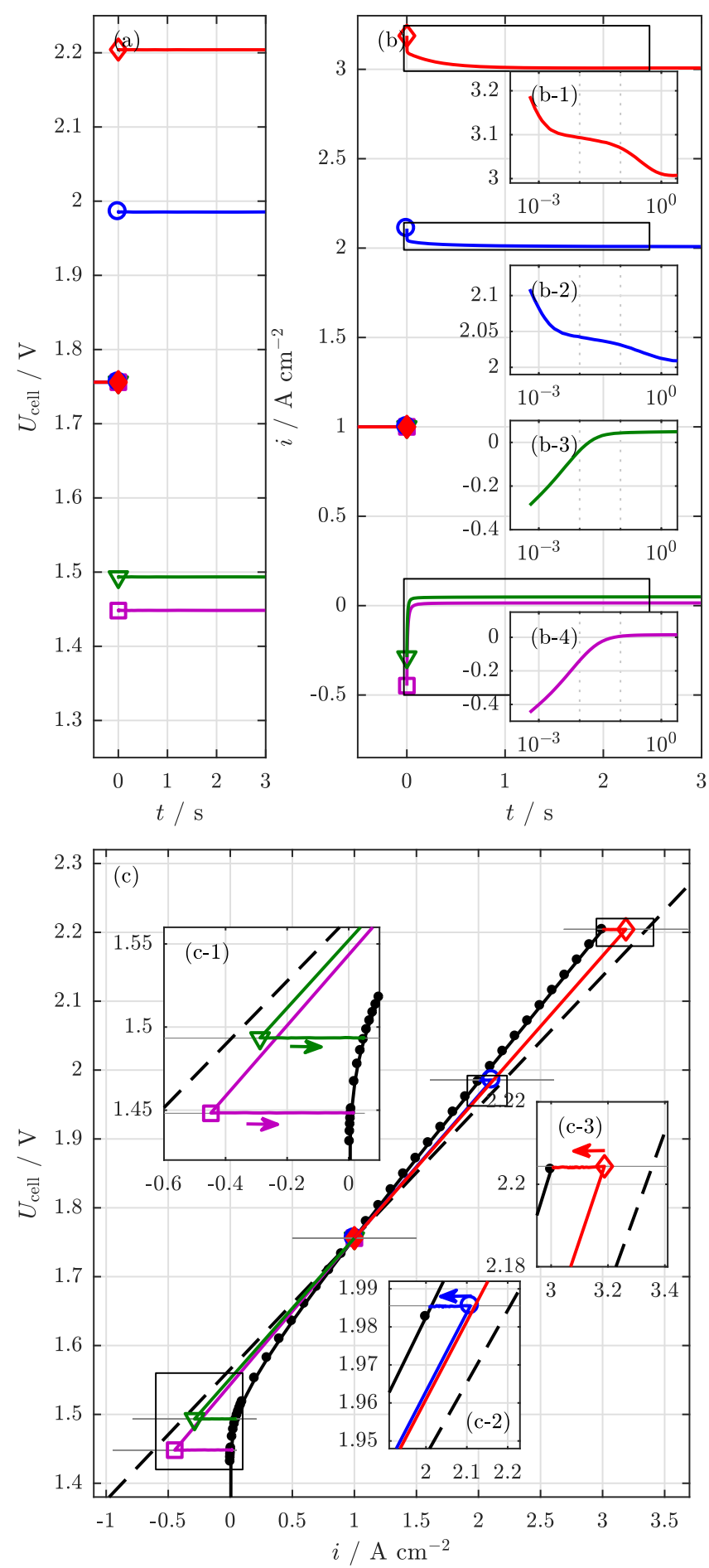

Figure 3. Voltage controlled upward and downward load steps; (a) Cell voltage input steps, full markers: start value, open markers: end value; (b) current density responses; Insets (b-1)-(b-4): different current density responses on a logarithmic time-scale. (c) steady state polarization curve (black dots) with trajectories of the four load steps in the Ui-phase space (with markers according to (a)).

points for upward steps, which is comparable to the galvanostatic upward steps. This suggests higher order dynamics for the voltage controlled upward steps as well, which cannot be clearly identified for the downward steps (Figs. 3b-3 and 3b-4).

Interestingly, for the downward cell voltage step, a negative current density response is measured initially, which reverses the current direction measured at the cell's current connection. For example, the 
observed cell voltage step from $1.756 \mathrm{~V}$ to $1.492 \mathrm{~V}$ shows an initial current density response of $-0.29 \mathrm{~A} \mathrm{~cm}^{-2}$. With time, the current density increases monotonously, intercepts the y-axis $\left(i=0 \mathrm{~A} \mathrm{~cm}^{-2}\right)$ after about $20 \mathrm{~ms}$ and reaches its new, positive steady state value $0.05 \mathrm{~A} \mathrm{~cm}^{-2}$ within $3.0 \mathrm{~s}$. Thus, the negative current density peak is six times higher than the expected steady state value. With regard to the downward step to $1.451 \mathrm{~V}$ (s. Fig. 3b-1), the negative peak current density is even lower $\left(-0.45 \mathrm{~A} \mathrm{~cm}^{-2}\right)$ and the ratio between steady state and peak value is higher by a factor of 45 .

Again, the time-dependent current density and cell voltage pairs are projected into the polarization space in Fig. 3c (colored, full lines). Analogous to the galvanostatic analysis, the black dots represent the steady state polarization curve and the horizontal, thin lines represent the start and the end voltages. The dashed line is calculated identically to the galvanostatic steps in Eq. 1 as linear equation with the slope of the area specific ohmic resistance in the starting point. It is shifted parallel, so that it intersects the starting point (analogous to the galvanostatic step in Fig. 2c and Galvanostatic steps section).

\section{Discussion}

In this section, the experimental results are discussed, starting with an analysis of the initial responses. The dynamic behavior is analyzed afterwards, followed by a detailed examination of the negative current densities in the potentiostatic mode. Finally, further aspects with regard to the experimental findings are discussed.

Initial response.-In Fig. $2 \mathrm{c}$ and Fig. $3 \mathrm{c}$ the steady state polarization curve is shown together with a dashed, linear curve, which intersects the staring point. This graph is well known from fuel cell literature ${ }^{11,13}$ and represents the locus of all points in the Ui-plane, which can be reached initially from the respective starting point. Zenith and Skogesdad ${ }^{11}$ presented this instantaneous characteristics as the algebraic part of the current-voltage-relation of a PEMFC. The ohmic losses of the cell do change initially with externally applied load changes, while all other processes of a cell change as continuous function.

A similar behavior can be seen with the PEMWE cell. As Fig. 2c and Fig. 3c show, the initial responses for all steps form a straight line with a similar slope as the instantaneous characteristic. The differences between the calculated dashed line and the measured initial responses occur due to imperfect input step signals. Especially, when the load steps exceed higher current or voltage ranges, the inputs are not ideal step signals but ramps, which do not fit perfectly to the calculated instantaneous locus. However, a qualitative accordance between the instantaneous characteristic calculated from the ohmic cell resistances and the measured initial response can be attested for all steps. A comparison of the load step types shows that a galvanostatic load step does not show any over- or undershoot in the cell voltage, while a potentiostatic step always leads initially to either an undershoot (downward) or an overshoot (upward) of the current density.

While this initial response behavior is well known for fuel cells ${ }^{13,19}$ to the best of our knowledge, the initial response behavior togehter with a current reversal has not been discussed for PEMWE. A first simplified analysis of the underlying effects will be given in this work in Current reversal and prototype model section. Before that, the dynamic behavior after the initial response of the system is investigated.

Dynamic behavior.-The second part of the transient behavior of the cell is the transition to a new steady state. Fuel cell literature has shown that transient load changes lead to initial answers, followed by various dynamic processes of higher orders. ${ }^{18,19}$ Experimental and modeling analyzes have shown several effects that can be attributed in general to activation and diffusion processes or the humidification of the membrane. ${ }^{13}$

As can be seen in the insets of Fig. 2 and Fig. 3, higher order dynamics can be observed for both types of upward steps equally in PEMWE. In comparison to Weydahl et al., ${ }^{19}$ who performed resistance controlled load steps for fuel cells, the trends of the dynamic response are qualitatively very similar. Quantitatively, they recorded relaxation times of $0.38 \mathrm{~ms}$ and $1.6 \mathrm{~ms}$ for the first dynamic process, which they attributed to activation processes, while a second relaxation time of $2 \mathrm{~s}$ for higher load steps corresponds most likely to diffusion effects. Response times of a similar order of magnitude are extracted from the upward load steps in the present analysis. In the inset of Fig. 3b-1 a first inflection point is located in the order of $\approx 10 \mathrm{~ms}$, a second one is in the order of magnitude of $\approx 300 \mathrm{~ms}$. With higher step sizes this second inflection point is more and more pronounced for both galvanostatic and potentiostatic load steps.

The dynamics of potentiostatic downward steps are of interest, because they can show temporary sign reversals, one initially and one during the run-in into a new steady state. In order to characterize and evaluate these negative currents, a simple model is presented in the following section.

Current reversal and prototype model.-Our experimental results show undershoots being typical for potentiostatic downward load steps and can also show a reversal of the current density, when the designated end cell voltage is below a certain level but still above the cell voltage for water splitting reaction. In order to describe the underlying processes and to evaluate the risks of a temporal current density reversal, a simple prototype model is presented.

The model includes the interaction between the HFR initial characteristics (s. Eq. 2) and the dynamic double layer charging and discharging as described by Zenith et al. ${ }^{11}$ (s. Eqs. 3 and 4). Additionally, the model includes a simplified description of the transport and temperature effects with a third dynamic equation (s. Eq. 5).

$$
\begin{aligned}
U_{\text {cell }}(t)= & U_{\text {rev }}^{0}(p, T)+\eta_{\mathrm{act}}^{\mathrm{a}}(t)-\eta_{\mathrm{act}}^{\mathrm{c}}(t) \\
& +\eta_{\mathrm{T}, \mathrm{conc}}^{\mathrm{a} / \mathrm{c}}(t)+i(t) \cdot r_{\mathrm{HFR}} \\
\delta^{\mathrm{a}} \cdot c_{\mathrm{dl}}^{\mathrm{a}} \frac{\mathrm{d} \eta_{\mathrm{act}}^{\mathrm{a}}}{\mathrm{d} t}= & +i(t)-i_{0}^{\mathrm{a}} \cdot\left[\exp \left(\frac{\alpha_{\mathrm{ox}}^{\mathrm{a}} \mathrm{F}}{\mathrm{R} T} \cdot \eta_{\mathrm{act}}^{\mathrm{a}}(t)\right)\right. \\
& \left.-\exp \left(-\frac{\alpha_{\mathrm{red}}^{\mathrm{a}} \mathrm{F}}{\mathrm{R} T} \cdot \eta_{\mathrm{act}}^{\mathrm{a}}(t)\right)\right] \\
\delta^{\mathrm{c}} \cdot c_{\mathrm{dl}}^{\mathrm{c}} \frac{\mathrm{d} \eta_{\mathrm{act}}^{\mathrm{c}}}{\mathrm{d} t}= & -i(t)-i_{0}^{\mathrm{c}} \cdot\left[\exp \left(\frac{\alpha_{\mathrm{ox}}^{\mathrm{c}} \mathrm{F}}{\mathrm{R} T} \cdot \eta_{\mathrm{act}}^{\mathrm{c}}(t)\right)\right. \\
& \left.-\exp \left(-\frac{\alpha_{\mathrm{red}}^{\mathrm{c}} \mathrm{F}}{\mathrm{R} T} \cdot \eta_{\mathrm{act}}^{\mathrm{c}}(t)\right)\right] \\
\tau \frac{\mathrm{d} \eta_{\mathrm{T}, \mathrm{conc}}^{\mathrm{a} / \mathrm{c}}}{\mathrm{d} t}= & -\eta_{\mathrm{T}, \mathrm{conc}}^{\mathrm{a} / \mathrm{c}}(t)+\eta_{\mathrm{T}, \mathrm{conc}}^{\mathrm{a} / \mathrm{sSt}}\left(i^{\mathrm{SSt}}\right)
\end{aligned}
$$

The cell voltage $U_{\text {cell }}(t)$ in Eq. 2 is described as the sum of the pressure and temperature corrected thermodynamic cell voltage $U_{\text {rev }}^{0}(p, T)$, the anode and cathode side activation overpotentials $\eta_{\text {act }}^{\mathrm{a}}(t)$, $\eta_{\text {act }}^{\mathrm{c}}(t)$, an additional temperature and concentration based overpotential $\eta_{\mathrm{T}, \mathrm{c} \text { conc }}^{\mathrm{a} / \mathrm{c}}(t)$ and the ohmic overpotential as the product of the current density and the corresponding area specific high frequency resistance $i(t) \cdot r_{\mathrm{HFR}}$. The dynamics of the double-layer are described separately for anode and cathode together with a description of the kinetics of each half-cell, based on the Butler-Volmer equation. In Eq. 3 and Eq. $4, c_{\mathrm{dl}}^{\mathrm{j}}$ represents the volume specific double layer capacitance, $\delta^{\mathrm{j}}$ the thickness of each catalyst layer, $i_{0}^{\mathrm{j}}$ the apparent exchange current densities, $\alpha_{o x}^{j}, \alpha_{\text {red }}^{j}$ the charge transfer coefficients, $R$ the ideal gas constant and $\mathrm{F}$ the Faraday constant. Eq. 5 describes the temporal change of the additional overpotentials. Here, $\eta_{\mathrm{T}, \text { conc }}^{\mathrm{a} / \mathrm{sSt}}\left(i^{\mathrm{SSt}}\right)$ represents the steady state voltage losses of the new steady state current density, $\tau$ is a fitted time constant. All parameters, constants, the boundary conditions and further used equations are shown in Appendix A. 

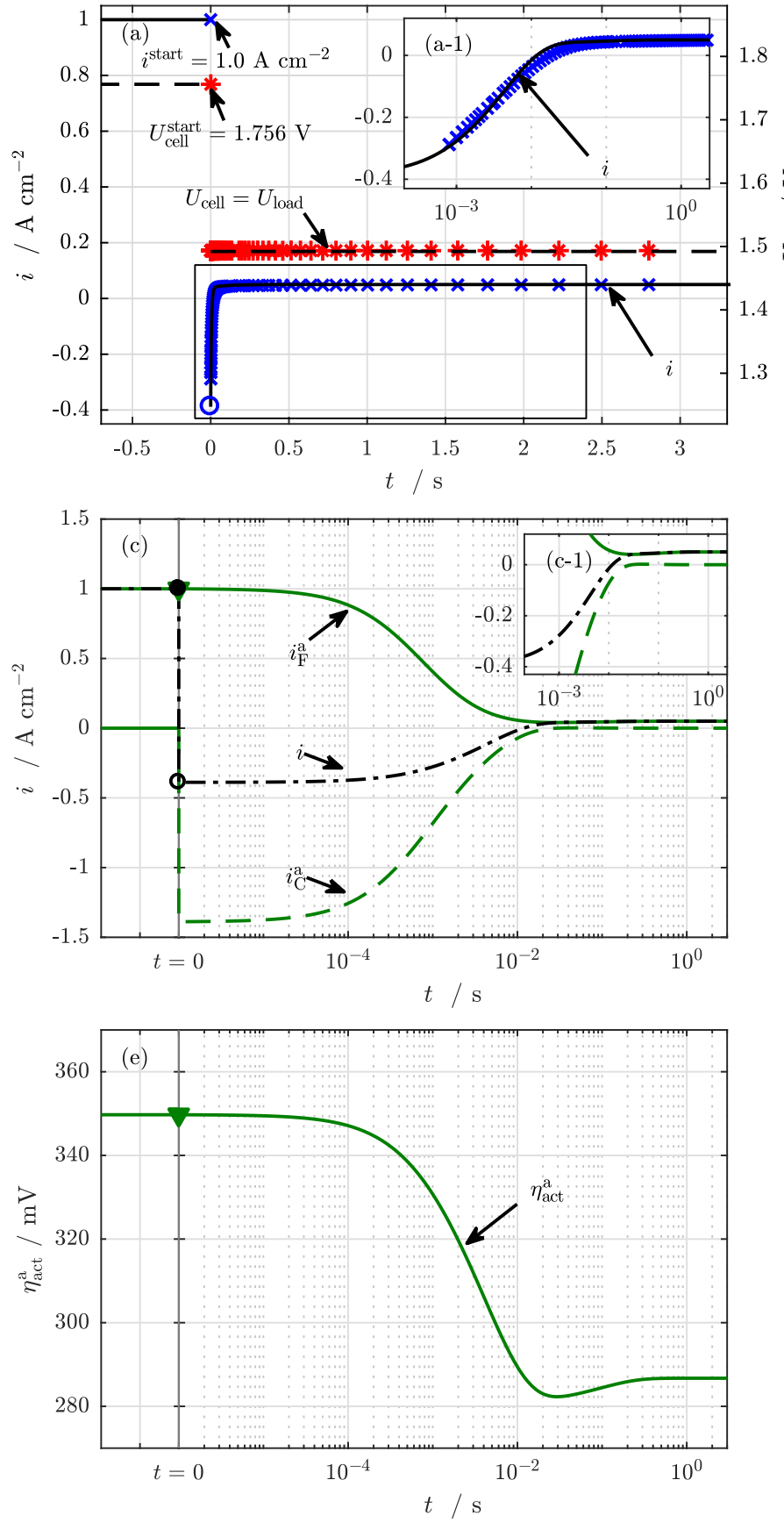
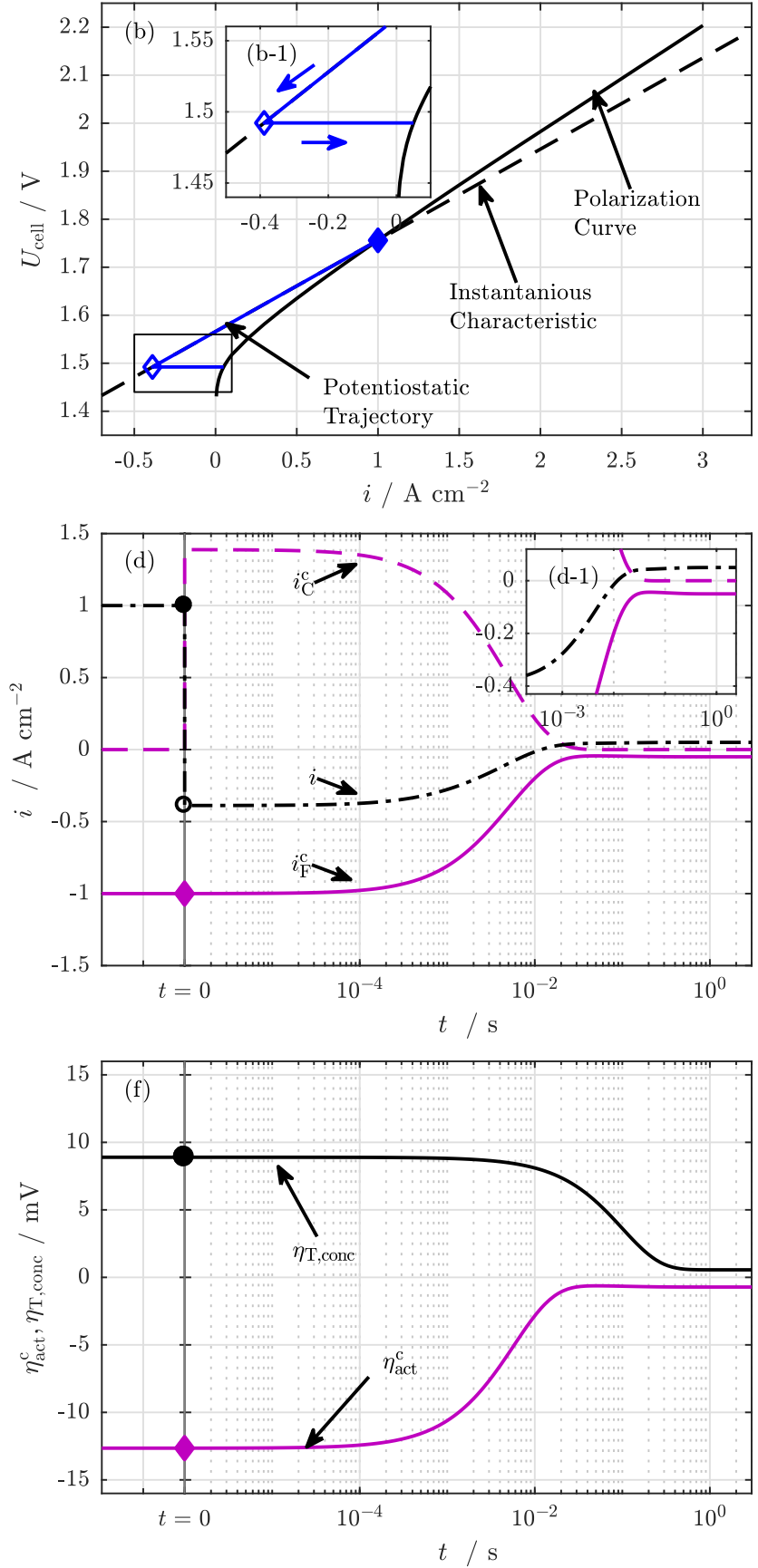

Figure 4. Simulation results for a potentiostatic step downwards; (a) the experimental current and voltage slopes, in accordance with the modeling results (a-1); (b) transformation of the modeling step in the Ui-phase space as trajectory; (c) internal current densities of the anode cell side, divided into capacitive and faradaic currents; (d) cathode side internal current densities, divided into capacitive and faradaic currents; (e) half-cell activation overpotential for anode; (f) half-cell activation overpotential for cathode and residual overpotential trends.

The model allows to perform different load step types. The following rules are given for voltage (s. Eq. 6a), current (s. Eq. 6b) and power controlled (s. Eq. 6c) steps:

$$
\begin{gathered}
0=U_{\text {cell }}-U_{\text {load }} \\
0=i \cdot A_{\text {cell }}-I_{\text {load }} \\
0=i \cdot A_{\text {cell }} \cdot U_{\text {cell }}-P_{\text {load }}
\end{gathered}
$$

Fig. 4 shows the simulation results of a potentiostatic downward step from $1.756 \mathrm{~V}$ to $1.492 \mathrm{~V}$. A comparison between the simulation and experimental results is given in Fig. $4 \mathrm{a}$, in which the input cell voltage and the output current density are presented over time. The dashed line represents the simulation input, the red stars show the corresponding experimental data. The simulated current density (solid, black line) and the experimental current density (blue crosses) show good qualitative and quantitative accordance on the linear scale but also on the logarithmic time scale (s. inset Fig. 4a-1). A maximal divergence between the simulation and the experimental results of $\Delta i_{\text {max }}=\left|i_{\text {exp }}-i_{\text {sim }}\right|=26 \mathrm{~mA} \mathrm{~cm}^{-2}$ is calculated.

As additional model validation, the potentiostatic upward step to $U_{\text {cell }}=2.204 \mathrm{~V}$ (corresponding to $3.0 \mathrm{~A} \mathrm{~cm}^{-2}$ ) is simulated. The upward step is analyzed in the appendix (Fig. A1) 
and shows a good qualitative accordance as well. Therefore, the model is considered as sufficiently reliable to perform further investigations.

The time-dependent current density and cell voltage trajectory are also transferred into the Ui-phase space. Fig. 4b shows that the initial response of the load step fits perfectly to the instantaneous characteristics related to the starting conditions, which is calculated with the experimental HFR. The comparison of the model and the experiment show that divergences between experimental initial response and instantaneous characteristics are highly influenced by uncertainties of the experimental input signal quality and the measurement accuracy.

Figs. $4 \mathrm{c}$ and $4 \mathrm{~d}$ shows the trend of the already discussed behavior of the integral current density (black, dash-dotted line) over time. Additionally, the faradaic (solid, colored lines) and the capacitive (dashed lines) current densities for anode (c) and cathode (d) are plotted. The faradaic current densities are the current density that correlate with the reaction rates of the half-cell reactions, whereas capacitive current densities charge and discharge the electrode double layers.

The capacitive current densities are zero under steady state conditions prior to the load step. Directly after the the downward step, the double layers charge or discharge with maximal rates and the capacitive current densities reach minimal/maximal values. Over time, they dynamically tend to zero again and reach a new steady state. The faradaic half-cell current densities instead monotonously decrease (anode) respectively increase (cathode) from their starting steady state to their new steady state condition, without any change of sign. During this run-in, the faradaic current densities are opposite to the corresponding capacitive current densities.

A similar trend is observed with the activation overpotential slopes in Figs. 4e and 4f) over time. The anode side activation overpotential is continuous (Fig. 4e, triangle) and remains positive during the dynamic step relaxation. The cathode activation overpotential (Fig. 4f, diamond) reduces continuously with no change of sign either. Together with the transient trend of the faradaic current densities this indicates that the directions of the electrolysis reaction on cathode and anode are not reversed but show reduced reaction rates in a downward step. The additional line shows the slope of all residual overpotentials for the downward step (Fig. 4f, circle), which shows a continuous trend as well. The residual overpotential shows an inflection point at $100 \mathrm{~ms}$, which is in the same order of magnitude as the second inflection point in the experimental data $(\approx 300 \mathrm{~ms}$, s. Fig. $2 \mathrm{~b}-1$ and Fig. $3 \mathrm{~b}-1)$. The time constant is fitted in the same order of magnitude $(\tau=100 \mathrm{~ms})$ to reach reasonable accuracy. This order of magnitude fits well to the hypothesis that the underlying processes are based on concentration and temperature effects.

Further aspects.-Even if the negative current densities may be uncritically for the electrochemical system, high negative current densities might increase the system complexity and become an issue for other system components such as the power electronics. As long as the instantaneous characteristic is a function of the ohmic resistance, it is worth to analyze the effect of different membrane thicknesses.

Fig. 5a shows the simulated polarization curves for different cell resistances (see full black Ui-lines) and the trajectories of potentiostatic downward steps. All steps are performed from the the cell voltages corresponding to $1.0 \mathrm{~A} \mathrm{~cm}^{-2}$ to cell voltages corresponding to $50 \mathrm{~mA} \mathrm{~cm}{ }^{-2}$ (colored lines). Additionally, the dashed line represents the polarization curve corrected by ohmic resistance.

The simulation results show on the one hand, the reduced ohmic resistances of the cell decrease the cell voltage. On the other hand, lower ohmic resistances intensify the negative current density peak. Theoretically, this peak can reach an infinite minimum, when there are no ohmic voltage losses, which can be seen in the inset of Fig. 5a-1. Here, all trajectories intersect at zero current density in a cell voltage of $U_{\text {cell }}\left(i=0 \mathrm{~A} \mathrm{~cm}^{-2}\right)=1.566 \mathrm{~V}$, which is identical to the iRfree cell voltage at the starting point $U_{\mathrm{iR}-\text { free }}\left(i=1.0 \mathrm{~A} \mathrm{~cm}^{-2}\right)$. With the recent trend to thinner membranes, ${ }^{5}$ the effect of negative current density peaks can be enhanced. Fig. 5 b shows how the negative current density peak intensifies, when the membrane thickness (and the ohmic
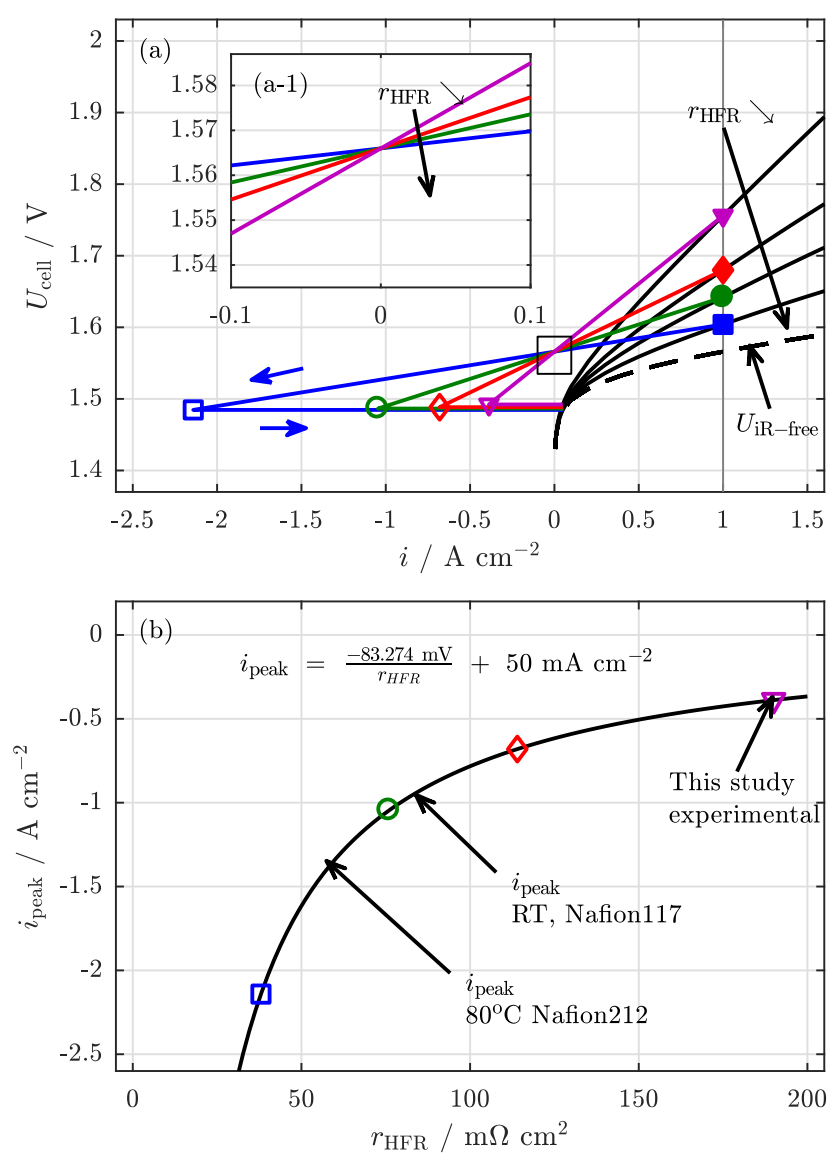

Figure 5. Simulation of reduced ohmic resistances (a) polarization curves (black full line) for different specific ohmic resistances $\left(38 \mathrm{~m} \Omega \mathrm{cm}^{2}\right.$, square; $76 \mathrm{~m} \Omega \mathrm{cm}^{2}$, circle; $114 \mathrm{~m} \Omega \mathrm{cm}^{2}$, diamond; $190 \mathrm{~m} \Omega \mathrm{cm}^{2}$, triangle), HFRcorrected polarization curve (dashed black line) and trajectories of potentiostatic downward steps (colored lines). full markers: starting value, open markers: end value; Inset (a-1) shows intercept of the trajectories. (b) Negative current density peaks calculated for the HFRs from (a) and calculated from literature data (for Nafion 117 from Ref. 20 and Nafion 212 from Ref. 21).

resistance) is reduced. For example, a reduction of the HFR down to $20 \%$ of the measured reference can lead to a negative current density peak of $i_{\text {peak }}=-2.1 \mathrm{~A} \mathrm{~cm}^{-2}$.

Additionally, the model allows to analyze the technically most relevant power controlled mode, in which current density and voltage show time-dependent response behavior. Fig. 6a shows a power step upwards (blue) and Fig. 6b downwards (red) from the reference starting point. The dashed lines represent the cell voltage, written on the right y-axis, the solid line shows the current densities on the left y-axis. The inset Fig. 6a-1 shows the cell voltage and current density response of the upward step, the inset Fig. 6b-1 shows the responses of the downward step, both on a logarithmic time scale. The upward step exhibits an overshoot in the current density similar to the voltage controlled step and a monotonous increase of the cell voltage like in the current controlled mode. Analogous, for the downward step a current density undershoot (similar to voltage controlled downward steps) and a monotonous voltage decrease (similar to current controlled downward steps) is observed.

This indicates that the power controlled mode is a combination of the potentiostatic and galvanostatic mode, with a superposition of both responses. The upward response shows dynamics of higher order, while the downward step always shows a current density undershoot. The main difference between the power controlled and the voltage controlled mode is that no negative current density peaks can occur, 

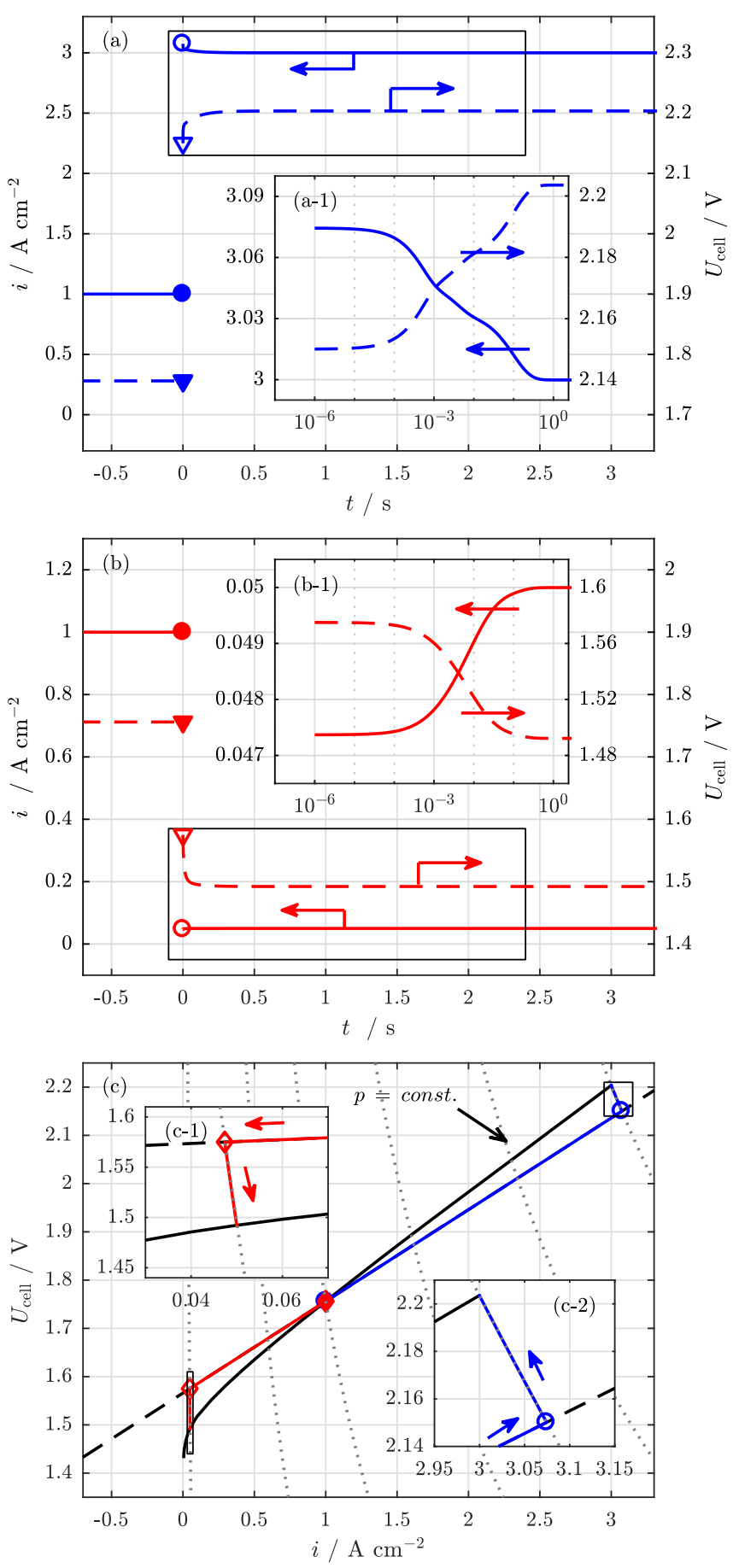

Figure 6. Simulation of constant power steps; current density (left y-axis, solid lines) and cell voltage (right y-axis, dashed lines) response for (a) an upward step (b) for a downward step; (c) steady state polarization curve (black) with trajectories of the two load steps in the Ui-phase space, with the insets (c-1) for the downward and (c-2) for the upward step; dotted lines represent lines of constant power.

which can be seen in the Ui-phase space of a power controlled step (s. Fig. 6c). Here, the trajectory of the load steps are shown, similar to Fig. 2c and Fig. 3c. Additionally, dotted gray lines of constant power are presented, which define the progression of the power responses. After the initial response, which follows the instantaneous characteristics from the HFR calculation, the dynamic run-in follows exactly the corresponding constant power line (s. inset of Fig. 6c-2). For very low power densities, the line of constant power is almost vertical in the observed region and shows therefore only minor undershoots in the current density, which can be seen in inset of Fig. 6c-1. As the constant power lines only occur in the first quadrant of the Ui-phase space, negative undershoots of current densities are impossible.

The simulation results show, that the power density step is not critical with regard to current or voltage reversal. However, upward steps show potentially high current overshoots, which must be considered technically.

\section{Conclusions}

Experimental load steps for a proton exchange membrane water electrolysis (PEMWE) cell were performed with a high temporally resolution in order to gain deeper insights into its dynamic behavior. Galvanostatic and potentiostatic steps were carried out, which revealed on the one hand a complex dynamic behavior especially with upward steps and on the other hand a current reversal for potentiostatic downward steps to low electrolysis cell voltages. To explain these phenomena proven analyzes from fuel cell literature are applied and a simple transient electrochemical PEMWE model is developed.

The comparison with fuel cell literature shows that current reversal effects are well known, especially, when a dynamic start-up is performed ${ }^{22}$ or the anode is exposed to hydrogen and oxygen simultaneously. ${ }^{23}$ Furthermore, voltage reversal ${ }^{24}$ is reported as well, in cases of hydrogen starvation on the anode side. Although these effects may not be stationary, they can significantly degrade the cell performance in fuel cells. ${ }^{25,26}$ Fuel cell literature explains that the fuel cell reversals is accompanied by a reversal or change of the reaction mechanisms in a majority of cases. In contrast, our experimental results do not indicate that these effects are relevant in the PEMWE.

Instead, a simple prototype model is capable to describe the dynamic behavior of load steps responses. The model results show that the half-cell potentials and the faradaic current densities of each halfcell remain in their conventional direction throughout the transient response without any change of sign. Consequently, it can be hypothesized that a temporal negative integral current density response is uncritical for the electrochemical behavior of the cell, because the internal processes, which are driven by the faradaic current densities, are not reversed during PEMWE load steps.

\section{Appendix}

In the following section additional model equations, parameters, assumptions, boundary conditions and a validation of the simulation results with experimental date is given, to strengthen the reliability of the model.

General model information and assumptions.-The polarization curve (s. Eq. 2) is calculated with the temperature and pressure corrected thermodynamic cell voltage. First, the temperature correction is realized with an empirical equation by Bratsch et al. ${ }^{27}$

$$
U_{\mathrm{rev}}^{0}(T)=1.2291 \mathrm{~V}-0.8456 \mathrm{mV} \cdot(T / \mathrm{K}-298.15)
$$

Furthermore, the pressure correction is implemented under the assumption of ideal gases and a fully saturated gas phase, which enables to calculate the activities of hydrogen and oxygen as the quotient of partial pressure and ambient pressure multiplied with an activity coefficient of $\gamma=1$ to $a_{\mathrm{H}_{2}}=a_{\mathrm{O}_{2}}=0.199$. The activity of liquid feed water was assumed as unity. ${ }^{27,28}$

$$
U_{\mathrm{rev}}^{0}(p, T)=U_{\mathrm{rev}}^{0}(T)+\frac{\mathrm{R} T}{2 \mathrm{~F}} \cdot \log \left(\frac{a_{\mathrm{H}_{2}} \sqrt{a_{\mathrm{O}_{2}}}}{a_{\mathrm{H}_{2} \mathrm{O}}}\right)
$$

Additionally, the kinetic equations (s. Eqs. 3,4) are simplified in this model. It is assumed, that the bulk concentration is identical to the concentration in the reaction zone, which means that no concentration effects are considered in the kinetics. The double layer capacity is distributed homogeneously in the catalyst layer and follows an ideal capacitance (s. Refs. 29,30).

All voltage losses, which cannot be attributed to ohmic or kinetic losses or the thermodynamic cell voltage are ascribed to the additional concentration and temperature based losses. These losses are fitted to the stationary solution of the polarization curve, the time constant is fitted to the experimental load data results. The model is isothermal, however, real temperature effects are mapped onto the additional voltage losses.

The model parameters are presented in Table A1. 
Table A1. Chosen parameters.

Parameter

Abbreviation

Value

Reference

Anode exchange current density

Cathode exchange current density

Charge transfer coefficient

Charge transfer coefficient

Charge transfer coefficient

Charge transfer coefficient

Temperature

Specific ohmic resistance

Specific anode capacitance

Specific cathode capacitance

Anode CL thickness

Cathode CL thickness

Time constant

Model validation.-To validate the model in addition to Current reversal and prototype model section, a potentiostatic upward step is simulated and compared to experimental data. Fig. A1 a shows the simulation results for a step from $1.756 \mathrm{~V}$ to $2.204 \mathrm{~V}$. The temporal evolution of the cell voltage input is identical for model (dashed line) and experiment (red stars). The current density instead, shows similar qualitative behavior for the simulation (full line) and the experiment (blue crosses). However, uncertainties in the quantitative behavior are observed in the inset Fig. A1a-1 on a logarithmic time scale. The differences between the experimental and the simulated current density progression indicate that the description of the temperature and concentration based voltage losses $\eta_{\mathrm{T}, \text { conc }}^{\mathrm{a} / \mathrm{c}}$ is too simplified, to fit the real trends for every kind of load step. Hence, the qualitative trend is reasonable and shows good accordance.
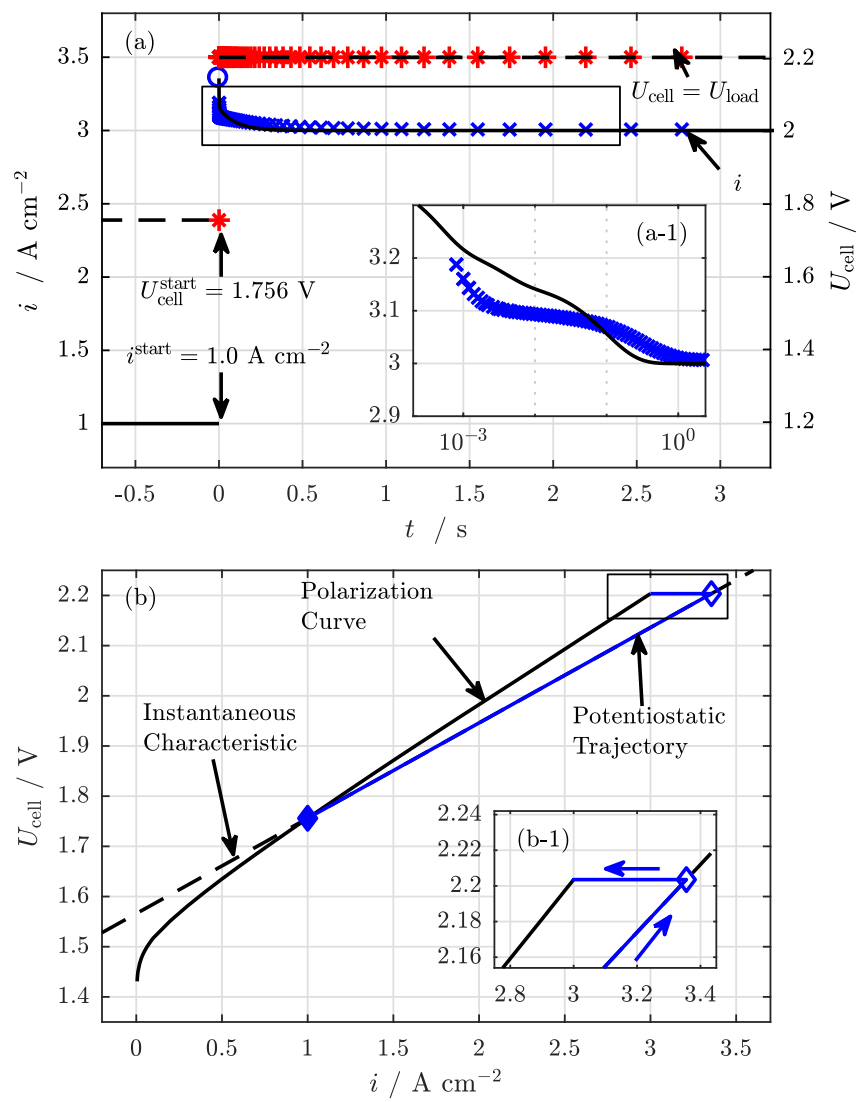

Figure A1. Simulation results for a potentiostatic upward step; (a) experimental current density and cell voltage slopes and (a-1) comparison with the modeling results; (b) transformation into the Ui-phase space as trajectory.

$$
\begin{gathered}
6 \times 10^{-8} \mathrm{~A} \mathrm{~cm}^{-2} \\
5 \times 10^{-1} \mathrm{~A} \mathrm{~cm}^{-2} \\
1.365 \\
1.365 \\
2 \\
2 \\
333 \mathrm{~K} \\
190 \mathrm{~m} \Omega \mathrm{cm}^{-2} \\
50 \mathrm{~F} \mathrm{~cm}{ }^{-3} \\
400 \mathrm{~F} \mathrm{~cm}^{-3} \\
10 \mu \mathrm{m} \\
15 \mu \mathrm{m} \\
0.1 \mathrm{~s}
\end{gathered}
$$

31

order of magnitude of Refs. 31,32

33

measured

measured

chosen between Refs. 34-36

35

for $\mathrm{IrO}_{2}$ electrode Ref. 37

Pt/C electrode; chosen between Refs. 38,39 fitted
Furthermore, Fig. A1b shows the Ui-phase space of the upward step. A very good accordance between the initial answer of the load step and the instantaneous characteristic can be seen here (s. Fig. Alb-1)

\section{ORCID}

C. Immerz (D) https://orcid.org/0000-0001-9163-0240

B. Bensmann (1) https://orcid.org/0000-0001-8685-7192

P. Trinke (D) https://orcid.org/0000-0002-0935-5321

M. Suermann (1) https://orcid.org/0000-0001-9685-7081

\section{References}

1. L. Allidières, A. Brisse, P. Millet, S. Valentin, and M. Zeller, Int. J. Hydrogen Energy, 44(20), 9690 (2019)

2. M. Kopp, D. Coleman, C. Stiller, K. Scheffer, J. Aichinger, and B. Scheppat, Int. J. Hydrogen Energy, 42(19), 13311 (2017).

3. H2Future Green Hydrogen, https://h2future-project.eu/ (accessed 17.06.2019).

4. HyBalance, http://hybalance.eu/. (accessed 17.06.2019).

5. U. Babic, M. Suermann, F. N. Büchi, L. Gubler, and T. J. Schmidt, J. Electrochem Soc., 164(4), F387 (2017).

6. P. Olivier, C. Bourasseau, and B. Bouamama, Int. J. Hydrogen Energy, 42(22), 14872 (2017).

7. H. Görgün, Int. J. Hydrogen Energy, 31(1), 29 (2006).

8. T. Yigit and O. F. Selamet, Int. J. Hydrogen Energy, 41(32), 13901 (2016)

9. J. Eichman, K. Harrison, and M. Peters, Novel Electrolyzer Applications: Providing More Than Just Hydrogen, National Renewable Energy Laboratory (2014).

10. D. Guilbert and G. Vitale, Energies, 12(4), 750 (2019).

11. F. Zenith, F. Seland, O. E. Kongstein, B. Børresen, R. Tunold, and S. Skogestad, J. Power Sources, 162(1), 215 (2006).

12. F. Zenith and S. Skogestad, J. Process Contr., 17(4), 333 (2007),

13. I. K. Peña Arias, P. Trinke, R. Hanke-Rauschenbach, and K. Sundmacher, Int. J. Hydrogen Energy, 42(24), 15818 (2017).

14. F. Jia, L. Guo, and H. Liu, Int. J. Hydrogen Energy, 39(24), 12835 (2014).

15. N. Zamel, R. Hanke-Rauschenbach, S. Kirsch, A. Bhattarai, and D. Gerteisen, Int. J. Hydrogen Energy, 38(35), 15318 (2013).

16. M. Suermann, T. J. Schmidt, and F. N. Büchi, Electrochim. Acta, 211, 989 (2016).

17. C. Immerz, B. Bensmann, P. Trinke, M. Suermann, and R. Hanke-Rauschenbach, $J$. Electrochem. Soc., 165(16), F1292 (2018).

18. K. P. Adzakpa, K. Agbossou, Y. Dube, M. Dostie, M. Fournier, and A. Poulin, IEEE Trans. Energy Convers., 23(2), 581 (2008).

19. H. Weydahl, S. Møller-Holst, G. Hagen, and B. Børresen, J. Power Sources, 171(2), 321 (2007).

20. L. Napoli, J. Franco, H. Fasoli, and A. Sanguinetti, Int. J. Hydrogen Energy, 39(16), 8656 (2014)

21. M. Bernt and H. A. Gasteiger, J. Electrochem. Soc., 163(11), F3179 (2016).

22. F. Jia, F. Liu, L. Guo, and H. Liu, Int. J. Hydrogen Energy, 41(15), 6469 (2016).

23. C. A. Reiser, L. Bregoli, T. W. Patterson, J. S. Yi, J. D. Yang, M. L. Perry, and T. d. Jarvi, Electrochem. Solid-State Lett., 8(6), A273 (2005).

24. P. Mandal, B. K. Hong, J.-G. Oh, and S. Litster, J. Power Sources, 397, 397 (2018).

25. A. Taniguchi, T. Akita, K. Yasuda, and Y. Miyazaki, J. Power Sources, 130(1-2), 42 (2004).

26. T. Engl, L. Gubler, and T. J. Schmidt, Energy Technology, 4(1),65 (2016).

27. S. G. Bratsch, J. Phys. Chem. Ref. Data, 18(1), 1 (1989).

28. D. R. Lide (Ed.), CRC Handbook of chemistry and physics: A ready-reference book of chemical and physical data, 85th ed., CRC Press, Boca Raton (2004). 
29. M. Ceraolo, C. Miulli, and A. Pozio, J. Power Sources, 113(1), 131 (2003).

30. P. Famouri and R. S. Gemmen, 2003 IEEE Power Engineering Society General Meeting, 13-17 July 2003.

31. K. W. Harrison, E. Hernández-Pacheco, M. Mann, and H. Salehfar, J. Fuel Cell Sci. Technol., 3(2), 220 (2006).

32. N. V. Dale, M. D. Mann, and H. Salehfar, J. Power Sources, 185(2), 1348 (2008).

33. J. Newman and K. E. Thomas-Alyea, Electrochemical Systems, 3rd ed., p. 8, WileyInterscience, Hoboken, N.J., (2004),
34. A. Kulikovsky, J. Electroanal. Chem., 823, 335 (2018).

35. D. Gerteisen, J. Electrochem. Soc., 162(14), F1431 (2015).

36. U. Babic, E. Nilsson, A. Pătru, T. J. Schmidt, and L. Gubler, J. Electrochem. Soc., 166(4), F214 (2019).

37. M. Bernt, A. Siebel, and H. A. Gasteiger, J. Electrochem. Soc., 165(5), F305 (2018).

38. Y. Liu, M. W. Murphy, D. R. Baker, W. Gu, C. Ji, J. Jorne, and H. A. Gasteiger, J. Electrochem. Soc., 156(8), B970 (2009).

39. T. Reshetenko and A. Kulikovsky, J. Electrochem. Soc., 165(5), F291 (2018). 